\title{
On the Paths of Categories
}

\author{
Kosta Došen
}

\begin{abstract}
To determine what deductions are it does not seem sufficient to know that the premises and conclusions are propositions, or something in the field of propositions, like commands and questions. It seems equally, if not more, important to know that deductions make structures, which in mathematics we find in categories, multicategories and polycategories. It seems also important to know that deductions should be members of particular kinds of families, which is what is meant by their being in accordance with rules.
\end{abstract}

Keywords Deduction - Proposition - Command - Question - Category • Multicategory $\cdot$ Polycategory $\cdot$ Rule $\cdot$ Natural transformation $\cdot$ Proof-theoretic semantics $\cdot$ General proof theory $\cdot$ Categorial proof theory

\section{Functions of Language}

In a terminology like that of the old logic, the notion of deduction will be for us primarily a hypothetical and not a categorical notion. (This use of categorical should not be confused with categorial, which is found later in this paper, and which, according to the Oxford English Dictionary [23], means "relating to, or involving, categories"; unfortunately, in mathematical category theory categorical dominates in the sense of categorial.) The distinction between categorical and hypothetical is found when we speak about categorical and hypothetical proofs. The latter is a proof under hypotheses, while the former depends on no hypothesis. Both may involve deduction, but we will be concerned here with deduction as found in hypothetical proofs.

Schroeder-Heister (together with P. Contu in [22], Sect. 4, in [20], Sect. 3, and in [21]; see also [8]) states that the reigning semantics — both classical semantics based on model theory and constructivist proof-theoretic semantics-is based on dogmas, the main one of which may be formulated succinctly by saying that categorical

\footnotetext{
K. Došen ( $\varangle)$

Faculty of Philosophy, University of Belgrade and Mathematical Institute, Serbian

Academy of Sciences and Arts, Knez Mihailova 36, p.f. 367, 11001 Belgrade, Serbia

e-mail: kosta@mi.sanu.ac.rs

(C) The Author(s) 2016

T. Piecha and P. Schroeder-Heister (eds.), Advances in Proof-Theoretic Semantics, Trends in Logic 43, DOI 10.1007/978-3-319-22686-6_4
} 
notions have primacy over hypothetical notions. We conform to this dogma when we take the notion of proposition, a categorical notion, to have primacy over the notion of deduction, a hypothetical notion. We conform to the same dogma when we take that, among functions of language, asserting, which is tied to propositions, is more basic than deducing.

The question for us here should not be what function of language is the most important in general, but what function of language is the most important for logic. Even if it were the case that asserting is the most important function of language in general, it could happen that, because of the specific goals it has, logic, though it takes into account the importance of asserting, gives precedence to the function of deducing. Even if asserting is the most important function of language in general, for a specific area another function may have precedence. In the nomenclature of a science wouldn't the most important function of language consist in naming rather than in asserting?

It is questionable however that there is a most important function of language in general. Following Frege's context principle from the introduction of the Grundlagen der Arithmetik "never to ask for the meaning of a word in isolation, but only in the context of a proposition" [14], and following the Wittgenstein of the Tractatus [25], as usually understood, the most important function of language should be asserting. The belief that there is such a function and that this function is asserting was however rejected by the Wittgenstein of the Philosophical Investigations [26]. The later Wittgenstein said that, using his terminology, there may be language games, appropriate to particular forms of life, where various functions of language like commanding, or questioning, would have precedence over asserting, and it could not be said that these language games are less fundamental. They are not meaningful only because behind them lurks somehow the activity of asserting.

Philosophers, scientists, those living a theoretical life, were inclined since ancient times to give precedence to naming, and more recently, as it happened with Frege and Wittgenstein in the Tractatus, they gave precedence to asserting. (The late Frege wanted to fuse the two activities.) But does language acquire meaning primarily in theoretical life? Are not the quarters where that life is led (something like a university campus, or a leisurely residential upper-class quarter) rather lately built and not central quarters in the city language (see [26], Sect. 18)?

Even though it is not essential to agree with the later Wittgenstein on this point, it helps to do so if we want to claim without worry that in logic the most important function of language is deducing. It is strange that one has to defend nowadays this rather venerable opinion, but so many developments in the philosophy of modern logic and the philosophy of language spoke against it in the last two centuries.

Textbooks of logic in the second half of the twentieth century would often start with a definition of logic as a human endeavour concerned with deduction, and would practically not mention deduction in the remainder of the book. It is only as the century was moving to its close that natural deduction or related matters started getting ground in textbooks of logic. 


\section{Deductions Not Necessarily Based on Propositions}

A deduction is usually taken to be a transition, a passage, from the premises to the conclusion. To simplify matters, let us assume that the premises, which are finitely many in numbers, have all been collected with the help of a connective like conjunction into a single one. Henceforth, until the last section, we will speak only about deductions that are a transition from one premise to one conclusion. Such deductions can mimic all the others.

The terms transition and passage in the preceding paragraph are far from being completely clear, and we shall return to them later, at the beginning of the third section. For the time being, let us concentrate on the premise and the conclusion. These are usually taken to be propositions, and by that is meant pieces of language that can be asserted. So it seems that our, rather common, characterization of deduction presupposes that we have propositions. Hence deducing presupposes asserting.

Could one imagine a deduction where one would pass from something that is not a proposition as a premise to something that is not a proposition as a conclusion? A deduction from a command to a command, or a deduction from a question to a question? Or, non-uniformly, a deduction from a proposition to a command, or from a command to a question? (In [24] and references therein one may find a defence of deductions where commands occur as premises and conclusions together with propositions. For the logic of questions, one may consult [16], and references therein; I don't know however of a reference dealing explicitly with deductions where questions occur as premises and conclusions, together perhaps with propositions or commands.)

Let us take a brief look at the uniform deductions, from a command to a command, or from a question to a question. Kolmogorov's contribution in [17] to the interpretation of intuitionistic logic that bears his name, besides those of Brouwer and Heyting, suggests that we should understand a deduction in constructive mathematics as taking us not from a proposition to a proposition, but from a problem to a problem. A problem however is something that does not seem to be necessarily tied with asserting. When the solution of a problem is expressed by a proposition, the statement of that problem may be a command, or a question. If the solution of our problem is "For $x, y$ and $z$ being respectively 3, 4 and 5 we have $x^{2}+y^{2}=z^{2}$ ", then the problem could be the command "Find three natural numbers $x, y$ and $z$ such that $x^{2}+y^{2}=z^{2}$ !" or the question "Are there three natural numbers $x, y$ and $z$ such that $x^{2}+y^{2}=z^{2} ?$ ?.

Kolmogorov's examples of problems in [17] are expressed by commands, but it seems that they could equally well be expressed by questions. It is not however clear in these examples that the solution should be expressed by a proposition rather than by producing one or several objects, i.e. by naming them.

From $x^{2}+y^{2}=z^{2}$ one can deduce $(z+x)(z-x)=y^{2}$. Can't we say therefore that from the command "Find three natural numbers $x, y$ and $z$ such that $x^{2}+y^{2}=z^{2}$ !" as a premise one can deduce the command "Find three natural numbers $x, y$ and $z$ such that $(z+x)(z-x)=y^{2}$ !" as a conclusion? Making one command would 
yield making the other. The second command would follow from the first, it could be inferred from it. And can't we say that from the question "Are there three natural numbers $x, y$ and $z$ such that $x^{2}+y^{2}=z^{2}$ ?" as a premise one can deduce the question "Are there three natural numbers $x, y$ and $z$ such that $(z+x)(z-x)=y^{2}$ ?" as a conclusion? Making one question would yield making the other. The second question would follow from the first, it could be inferred from it.

To make such a deduction with commands it is not necessary to assume that the command in the premise is actually made, as in deductions with propositions it is not necessary to assume that the premise is actually asserted. Analogously, to make such a deduction with questions it is not necessary to assume that the question in the premise is actually put.

To make a deduction with propositions it does not matter whether the premise is true or not. The premise being false does not invalidate the deduction. It would invalidate it as a proof, if the deduction was proposed as a proof of the conclusion. As a deduction simpliciter, it is however perfectly legitimate with a false premise. Analogously, to make a deduction with commands it would not matter whether the premise can be fulfilled or not. The premise being impossible to fulfil would not invalidate the deduction. With propositions the deduction may serve to show that the premise is false because it yields a false conclusion, as in reductio ad absurdum. With commands, the deduction might serve to show that the premise cannot be fulfilled, because it yields a conclusion that cannot be fulfilled.

Commands here are assumed to have two fulfilment values: can be fulfilled and cannot be fulfilled, but it is not clear that therefore the logic of commands should be taken as fulfilment-functional and two-valued. The negation of a problem $p$ need not be interpreted as it is not possible to fulfil $p$, but as from the assumption that $p$ can be fulfilled one can derive a contradiction, which is in tune with intuitionistic logic (see [17]). The implication of that logic may be tied to deduction, and hence it would be intuitionistic.

Can the notion of deduction be widened so as to cover also non-uniform deductions involving propositions, commands and questions, like those mentioned above? Not all of these deductions need make sense. It is indeed not easy to see what would be a deduction from a command to a question. It could however again be a transition from a problem to a problem, as suggested by Kolmogorov. Deductions from propositions to commands, and vice versa, from commands to propositions, are easier to conceive, and have been examined in [24].

We shall next consider a matter that would extend even more the range of the application of the word deduction, and go beyond the linguistic sphere. We would thereby transcend its widest application in this sphere.

Can one make deductions involving non-linguistic entities as premises or conclusions? Could one take as a premise the perception of something small $a$ and something big $b$, and deduce from that as a conclusion the proposition that $a$ is smaller than $b$ ? Can this transition from a perception to a proposition be called a deduction? And can one deduce from a proposition a perception, not of something external, but a mental image? And can one deduce one mental image from another mental image? Why should this widening of the application of the word deduction to the non-linguistic 
sphere represent a danger for the mathematical theory of deduction, which it is the duty of logic to formulate and investigate, and which we will consider in the next two sections?

We will not go so far as to claim that the premise and conclusion of a deduction can be anything. It seems that one could take a name as a premise or a conclusion of a deduction only in an elliptical sense. From the context one can find the proposition involving the name for which the name stands. Without that context, from a pure name, it is not clear that one could deduce anything. (Kolmogorov's solutions that are objects, which we mentioned above, could be taken as being solutions in an analogous elliptical sense.)

It does not seem unreasonable to claim however that premises and conclusions can be other things than propositions. Formulae with free variables are tied to propositions, but are not strictly speaking propositions. ${ }^{1}$ These things, and things like commands and questions, may perhaps be tied in some way with propositionsthough they are not propositions, they are somehow in the same field. On the other hand, the connection with propositions in the case of perceptions and mental images becomes less clear. Are they too in the field of propositions?

\section{Deductions in Categories}

One can surmise the following. The specificity of transitions that are deductions is not made uniquely of the things these transitions connect. Deductions are not singled out by specifying what can be premises and conclusions. Something having to do with these transitions themselves, independently of the premises and conclusions, determines that we have to do with deductions.

What could that be? What are anyway the transitions, the passages, that deductions are? Is the active, dynamic, component in the word transition essential?

One can next surmise the following. The specificity of transitions that are deductions does not consist in this active component. That side of the matter is psychological and is not essential from a mathematical, and logical, point of view. (The dangers of psychologism that lurk here are considered in [8].) Reified in mathematics, deductions are like arrows in a category.

A category is made of a class whose elements are called arrows, and another class whose elements are called objects, and two functions from arrows to objects-one that assigns to every arrow an object that is its source, and the other that assigns to every arrow an object that is its target. We also have operations on arrows, about which we will speak in a moment. Otherwise, arrows are not specified more closely. They can be anything, provided they have sources and targets, and the required operations. The notion of arrow is very abstract, like the notion of point or the notion of line in geometry. It is anything that satisfies the assumptions, which are very abstract too.

\footnotetext{
${ }^{1}$ I am grateful to Thomas Piecha for suggesting this.
} 
In the same way, deductions have a source, called a premise, and a target, called a conclusion, and they make a structure given by operations on them. It happens that on deductions such as we have envisaged them here, with a single premise and a single conclusion, the main operations are exactly like the main operations on arrows in categories.

These are the operations that enter into the definition of a category, and they are the binary operation of composition

$$
\frac{f: A \rightarrow B \quad g: B \rightarrow C}{g \circ f: A \rightarrow C}
$$

which in terms of deductions is a simple form of cut of sequent systems, and the nullary operations of identity arrows $\mathbf{1}_{A}: A \rightarrow A$, which as deductions are the trivial identity deductions where the premise and conclusion coincide-the primordial deductions, the axiomatic sequents. The operation of composition is partial; the target of $f$ must be the source of $g$ for $g \circ f$ to be defined.

For categories, one assumes associativity of composition:

$$
\begin{gathered}
\frac{f: A \rightarrow B \quad g: B \rightarrow C}{g \circ f: A \rightarrow C} \quad h: C \rightarrow D \\
h \circ(g \circ f): A \rightarrow D \\
\frac{g: A \rightarrow B \quad \frac{g: B \rightarrow C \quad h: C \rightarrow D}{h \circ g: B \rightarrow D}}{(h \circ g) \circ f: A \rightarrow D} \\
h \circ(g \circ f)=(h \circ g) \circ f,
\end{gathered}
$$

which makes perfect sense as an equality of deductions-it is about permuting cut with cut in sequent systems. This permuting is involved in usual cut elimination procedures (see [1], Sect.2), and less usual ones (see [4], Chap. 1). It is however interesting in its own right, independently of these procedures. It is a perfectly natural assumption about deductions, with which they make the deepest kind of mathematical structure - a structure one finds in all categories, and in particular in the category of sets (which we will consider below).

Let us note that if, as in the Curry-Howard correspondence, one designates deductions by typed lambda terms, which is congenial with understanding proofs in the categorical, and not the hypothetical, i.e. categorial, way (see [8], Sect. 4), then composition of deductions is represented by substitution. With that, associativity of composition becomes invisible, unless one introduces, as it is sometimes done, an explicit substitution operator (see [19]). This unary operator is obtained by currying binary composition. Instead of $g \circ f$, we have something like $g\langle x, f\rangle$, which corresponds to " $g$ where for $x$ one substitutes $f$ ", and where $\langle x, f\rangle$ is a unary operator applied to $g$. Analogously, $h\langle y, g\rangle$ corresponds to " $h$ where for $y$ one substitutes $g$ ", and associativity of composition becomes 


$$
h\langle y, g\langle x, f\rangle\rangle=h\langle y, g\rangle\langle x, f\rangle .
$$

It does not seem we will get closer to associativity with other notations for explicit substitution (like, for example, the notation with inverse order suggested by [13], where $g\langle x, f\rangle$ is replaced by something like $\langle f, x\rangle g$, and our equation becomes $\langle\langle f, x\rangle g, y\rangle h=\langle f, x\rangle\langle g, y\rangle h$, or a vertical notation like $g_{f}^{x}$, with which our equation becomes $\left.h_{g_{f}^{x}}^{y}=h_{g f}^{y x}\right){ }^{2}$ It is improbable that one could have reached the notion of category, and realized its importance, by conceiving and representing matters pertaining to composition in that manner.

In categories one assumes moreover identity laws, i.e. laws of composing with identity arrows:

$$
\begin{gathered}
\frac{\mathbf{1}_{A}: A \rightarrow A \quad f: A \rightarrow B}{f \circ \mathbf{1}_{A}: A \rightarrow B} \\
\frac{\mathbf{1}_{B}: B \rightarrow B}{f: A \rightarrow B \quad B} \\
\mathbf{1}_{B} \circ f: A \rightarrow B \\
f \circ \mathbf{1}_{A}=\mathbf{1}_{B} \circ f=f,
\end{gathered}
$$

which in terms of deductions say that composing a deduction with an identity deduction, either on the side of the premise or on the side of the conclusion, leaves the deduction unchanged. This again makes perfect sense as an equality of deductions, and is an essential ingredient of cut elimination. When the cuts have been pushed to the top of the derivation, where they are performed with axiomatic sequents, they disappear.

Lambek (see [18]) called deductive system the notion generalizing categories by not assuming the associativity of composition and the laws of composing with identity arrows. In [3] and [4] (Sect. 1.9) one can see how this notion of deductive system is characterized proof-theoretically by a representation result in the style of Stone, and how the notion of category is characterized proof-theoretically by a representation result in the style of Cayley.

There may be further operations on arrows, with which we enter into the field of categories with additional structure. One such operation is tied to the biendofunctor of product, which corresponds to conjunction, both in classical and intuitionistic logic. Coproduct, with which another such operation is tied, corresponds to disjunction, in both logics again. With product and coproduct we obtain equations between deductions that make perfect sense in proof theory. They stem from adjointness of functors, and are related to normalization in natural deduction and cut elimination in sequent systems (see [4], [5] and [9]). Other equations, like those involving distributivity of product over coproduct, i.e. conjunction over disjunction, may, but need not, be based on adjointness. Intuitionistic logic will differ essentially from classical logic by tying implication to adjointness (see [2] and [4]), which should not be done for

${ }^{2}$ Roy Dyckhoff was kind to comment upon this. 
classical, material, implication (see [9], Chap. 14). We will not go further into categorial proof theory, which deals with the equations between deductions suggested by such categories with additional structure. Let it be said only that it is remarkable how equations important in mathematics in general, or in particular fields of mathematics, reemerge as perfectly sensible equations between deductions (see [7]).

What was surmised above is that the structure that deductions make with such operations is an essential ingredient in the notion of deduction. Could one go as far as to take this as the main ingredient? As in category theory, the structure of arrows would be the main thing. And, as in category theory, the arrows would be more important than the objects. With deductions the objects are the premises and conclusions, and these premises and conclusions, whatever they are preciselypropositions, commands, questions, problems, or something else-would not precede the arrows. Deducing would not be preceded by asserting, or another function of language.

When functions are reified as sets of ordered pairs, the active, dynamic, component in the notion of function is lost. This component, which comes from psychology, is also lost in the reification brought by the categorial notion of function, where a function is an arrow in a category. Categorially, functions in the category Set, where the objects are sets and the arrows are functions, are characterized through composition and identity functions. The same operations characterize deductions in general.

Although Set has the structure that deductions make, it is not natural for its objects to be called premises and conclusions, and for its arrows to be called deductions. One reason may be the nature of these objects, which are not in the field of propositions (see the end of the preceding section). Another reason might be that we have too many of these deductions. Any two objects would be connected by a deduction, except when the premise is not empty while the conclusion is empty. Deductions, in the categories where arrows may be more naturally designated by that term, are usually more discriminatory. There are more objects not connected by arrows.

The structure of deductions imitates the structure of the category Set even more when they involve the binary connectives of conjunction, disjunction and implication, together with the nullary connectives $T$ and $\perp$. This structure, appropriate for intuitionistic propositional logic, imitates Set with the biendofunctors of product, coproduct and exponentiation (for exponentiation we have covariance in the base and contravariance in the exponent), together with the terminal and initial objects, i.e. a singleton and the empty set. Still, the arrows of the category Set could not be taken as deductions, but only as their model. (The question of models of deductions was discussed in [6].) This is the model that stands behind the standard proof-theoretic semantics for intuitionistic logic, which through the Curry-Howard correspondence is tied to the typed lambda calculus.

Matters become clearer when in conceiving this semantics we do not conform to the dogma mentioned at the beginning. When we look upon this semantics hypothetically, and not categorically, as in the typed lambda calculus, we will end up in the categorial setting of Set. With the typed lambda calculus we also end up in the sets of Set, but the categorial setting is hidden. 
In [9] one may find a categorial setting for the proof theory of classical conjunctivedisjunctive logic different from that of Set, which leads to a categorial setting for the proof theory of the whole of classical propositional logic where a characterization through adjunction for classical implication is relinquished. Implication is again characterized through adjunction in the categorial setting for the proof theory of linear propositional logic without modalities, which is investigated in [10].

\section{Deductions in Multicategories and Polycategories}

Let us consider now the deductions where we can have more than one premise, though we still have a single conclusion. Such deductions, which correspond to Gentzen's singular sequents, with not more than one formula on the right-hand side, correspond to arrows $f: \Gamma \rightarrow A$ in Lambek's multicategories, sometimes called multiarrows, where capital Greek letters like $\Gamma$ stand for finite sequences of objects. A particular kind of multicategory is an operad, where $\Gamma$ in multiarrows $f: \Gamma \rightarrow A$ is a finite sequence every member of which is $A$. The algebraic notion of operad, which has arisen in algebraic topology, has been much investigated lately. (References concerning the notions of operad and multicategory, and a discussion of matters concerning them, may be found in [11].)

Multicategories can be mimicked by categories with additional structure like monoidal categories, which have a binary operation on objects like conjunction, enabling us to bind the objects in $\Gamma$ into a single object. The particular structure of multicategories is however important and interesting, and we shall now examine one aspect of it.

In multicategories instead of composition we have insertion operations on multiarrows:

$$
\frac{f: \Gamma \rightarrow A \quad g: \Delta, A, \Theta \rightarrow B}{g \triangleleft f: \Delta, \Gamma, \Theta \rightarrow B}
$$

which correspond to Gentzen's cut of singular sequents. The notation $g \triangleleft f$ is ambiguous, because it does not specify the cut formula. This ambiguity is remedied with the more precise notation of [11], but for the comments we will make here we can do with the less precise notation we have just introduced.

In multicategories, besides the associativity of insertion that corresponds to the associativity of composition in categories:

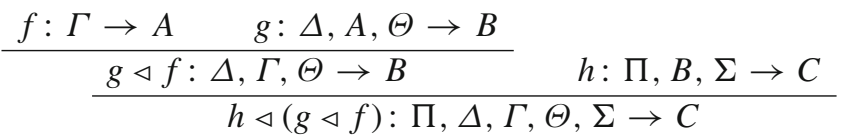

$$
\begin{aligned}
& \frac{f: \Gamma \rightarrow A \quad \frac{g: \Delta, A, \Theta \rightarrow B \quad h: \Pi, B, \Sigma \rightarrow C}{h \triangleleft g: \Pi, \Delta, A, \Theta, \Sigma \rightarrow C}}{(h \triangleleft g) \triangleleft f: \Pi, \Delta, \Gamma, \Theta, \Sigma \rightarrow C}
\end{aligned}
$$




$$
h \triangleleft(g \triangleleft f)=(h \triangleleft g) \triangleleft f,
$$

we have another kind of associativity of insertion, which involves also commutativity:

$$
\begin{aligned}
& \frac{g: \Sigma \rightarrow B \quad \frac{f: \Gamma \rightarrow A \quad h: \Delta, A, \Theta, B, \Pi \rightarrow C}{h \triangleleft f: \Delta, \Gamma, \Theta, B, \Pi \rightarrow C}}{(h \triangleleft f) \triangleleft g: \Delta, \Gamma, \Theta, \Sigma, \Pi \rightarrow C} \\
& \frac{f: \Gamma \rightarrow A \quad \frac{g: \Sigma \rightarrow B \quad h: \Delta, A, \Theta, B, \Pi \rightarrow C}{h \triangleleft g: \Delta, A, \Theta, \Sigma, \Pi \rightarrow C}}{(h \triangleleft g) \triangleleft f: \Delta, \Gamma, \Theta, \Sigma, \Pi \rightarrow C} \\
& (h \triangleleft f) \triangleleft g=(h \triangleleft g) \triangleleft f,
\end{aligned}
$$

This other associativity is interesting algebraically and combinatorially. It is also related to interesting polyhedra (see [11], Sect. 13).

In polycategories we have arrows $\Gamma \rightarrow \Delta$, which correspond to the plural sequents of Gentzen, where there may be several formulae on the right-hand side too. The arrows of polycategories correspond to the deductions of classical logic, which are investigated graph-theoretically in [12]. For polycategories and their operations of insertions, which correspond to Gentzen's cut for plural sequents, we have besides the associativity and the associativity involving commutativity on the lefthand side, analogous to those we have just given for multicategories, another associativity involving commutativity on the right-hand side (see [12], Propositions 2.1-3).

These plural deductions of classical logic are not very natural. They have been invented following Gentzen's suggestion, and not found by describing deduction in real life. They provide however the best means to understand classical logic prooftheoretically. They are implicit in the categorial approach to the proof theory of classical logic of [9], and in the categorial approach to the proof theory of linear logic of [10].

The remarks made here on deduction lead to the following tentative characterization of this notion. A deduction is an arrow in a category, a multicategory, or a polycategory, where the objects are more or less akin to propositions-something in the field of propositions.

\section{Rules for Deductions}

Nothing has been said up to now about deductions being in accordance with rules. When we deal with formal deductions, i.e. the deductions of logic, and they are conceived as arrows in categories with additional structure, which is brought by something like the functors corresponding to connectives that we mentioned in the third section, then our deductions are members of families of arrows indexed by 
the objects of the category, which are usually natural transformations involving the functors we have just mentioned. Being in accordance with rules here amounts to being members of such families, and the schematic character of the rules is given by the indexing by objects of the members of our families of arrows. With this indexing, the objects that are indices serve to make the sources or targets of the arrows, i.e. the premises or conclusions of the deductions. For example, the natural transformation $p^{1}$ of the first projection for conjunction elimination with the indices being the objects $A$ and $B$ gives the deduction $p_{A, B}^{1}: A \wedge B \rightarrow A$.

Is this indexing necessary for the notion of rule for deduction? Can a rule correspond to a family of arrows that is a singleton, without indexing? Can one call rule something which covers a single deduction, with which a single deduction is in accordance? In or outside logic, is generality necessary for rules? Should a rule always cover many cases? Can a rule cover a single case?

Deductions that are not in logic may still resemble the formal deductions of logic by being in accordance with schematically given rules. Such would be the deduction from the premise "The day before yesterday was Thursday" to the conclusion "Tomorrow will be Sunday" (though it is not immediately clear how to formulate the rules in question). If they cannot be found in logic, could one find outside logic deductions that are not instances of something schematic? Shouldn't they be in accordance with rules? What would be the appropriate notion of rule there? When bereft of its psychological or sociological aspects, like compulsoriness, would this notion of rule leave something to be investigated by precise, perhaps even mathematical, means? Grammar and linguistics may give an inspiration for considering such matters, which are close to the concerns of the later Wittgenstein.

In [27] (end of Lecture XIII, Lent Term 1935) Wittgenstein taught that "a rule is something applied in many cases", but then disparaged this remark off-handedly. He considered it useless for learning how to use a rule. Why must this remark serve that purpose? In another context, where the purpose is to explain what rules are and not to teach how to use them, it may prove important to determine whether generality is necessary for rules. Wittgenstein returned to this question in [26] (Sect. 199) and in other places (for references see [15], Sect. 199, pp. 120-124), with consideration towards the generality of rules, which he put within a wider scheme.

Wittgenstein ended the lecture from which we have quoted above by a nice and enigmatic picture: "A rule is best described as being like a garden path in which you are trained to walk, and which is convenient." A path is usually something taken many times, by many people. If a rule is like a path, the deductions in accordance with the rule could perhaps be like many particular walks on this path. We will however try to consider more closely this and other matters mentioned in this section on another occasion.

Acknowledgments Work on this paper was supported by the Ministry of Education, Science and Technological Development of Serbia, while the Alexander von Humboldt Foundation has supported the presentation of a part of it at the Second Conference on Proof-Theoretic Semantics, in Tübingen, in March 2013. I am grateful to the organizers of that conference, and in particular Peter SchroederHeister, for their exceptional hospitality. I am also grateful to him and to Thomas Piecha for making some useful comments on this paper. 
Open Access This chapter is distributed under the terms of the Creative Commons Attribution Noncommercial License, which permits any noncommercial use, distribution, and reproduction in any medium, provided the original author(s) and source are credited.

\section{References}

1. Borisavljević, M., Došen, K., Petrić, Z.: On permuting cut with contraction. Math. Struct. Comput. Sci. 10, 99-136 (2000). http://arXiv.org

2. Došen, K.: Deductive completeness. Bull. Symb. Log. 2(523), 243-283 (1996). For corrections see [4], Section 5.1.7, and [5]

3. Došen, K.: Deductive systems and categories. Publications de l'Institut Mathématique N.S. 64(78), 21-35 (1998)

4. Došen, K.: Cut Elimination in Categories. Kluwer, Dordrecht (1999)

5. Došen, K.: Abstraction and application in adjunction. In: Kadelburg, Z. (ed.) Proceedings of the Tenth Congress of Yugoslav Mathematicians, pp. 33-46. Faculty of Mathematics. University of Belgrade, Belgrade (2001) Available at: http://arXiv.org

6. Došen, K.: Models of deduction. In: Kahle, R., Schroeder-Heister, P. (eds.) Proof-Theoretic Semantics, Proceedings of the Conference "Proof-Theoretic Semantics, Tübingen, 1999", Synthese, vol. 148, pp. 639-657 (2006). Available at: http://www.mi.sanu.ac.rs/kosta/publications. htm

7. Došen, K.: Algebras of deductions in category theory. In: Jokanović et al. (eds), Third Mathematical Conference of the Republic of Srpska, Proceedings, Trebinje 2013, Zbornik radova, vol. I, pp. 11-18. Univerzitet u Istočnom Sarajevu, Fakultet za proizvodnju i menadžment, Trebinje (2014). Available at: http://www.mi.sanu.ac.rs/kosta/DosenAlgebrasofDeductions.pdf; http://www.mk.rs.ba/wp-content/uploads/2015/02/TOM1-Copy.pdf, pp. 1-8

8. Došen, K.: Inferential semantics. In: Wansing, H. (ed.) Dag Prawitz on Proofs and Meaning, pp. 147-162. Springer, Cham (2015). Preprint of 2012 available at: http://www.mi.sanu.ac.rs/ kosta/publications.htm

9. Došen, K., Petrić, Z.: Proof-Theoretical Coherence. KCL Publications (College Publications), London (2004). Revised version of 2007 available at: http://www.mi.sanu.ac.rs/kosta/ publications.htm

10. Došen, K., Petrić, Z.: Proof-Net Categories. Polimetrica, Monza (2007). Preprint of 2005 available at: http://www.mi.sanu.ac.rs/kosta/publications.htm

11. Došen, K., Petrić, Z.: Weak cat-operads. (2010). Available as v8, the last version of the authors, at: http://arXiv.org

12. Došen, K., Petrić, Z.: Graphs of plural cuts. Theor. Comput. Sci. 484, 41-55 (2013). Available at: http://arXiv.org

13. Dyckhoff, R., Pinto, L.: Cut-elimination and a permutation-free sequent calculus for intuitionistic logic. Studia Logica 60, 107-118 (1998)

14. Frege, G.: Die Grundlagen der Arithmetik: Eine logisch mathematische Untersuchung über den Begriff der Zahl. Verlag von Wilhelm Koebner, Breslau (1884). English translation by Austin, J.L.: The Foundations of Arithmetic: A Logico-Mathematical Enquiry into the Concept of Number, 2nd revised edn. Blackwell, Oxford (1974)

15. Hacker, P.M.S.: Wittgenstein: Rules, Grammar and Necessity-Essays and Exegesis of $\S \S 185-$ 242. Wiley-Blackwell, Chichester (2009). An Analytical Commentary on the Philosophical Investigations, 2nd extensively revised edn. (1st edn. of 1985 by Baker, G.P., Hacker, P.M.S.)

16. Harrah, D.: The logic of questions. In: Gabbay, D., Guenthner, F. (eds.) Handbook of Philosophical Logic, vol. 8, pp. 1-60. Kluwer, Dordrecht (2002)

17. Kolmogorov, A.N.: Zur Deutung der intuitionistischen Logik. Mathematische Zeitschrift 35, 58-65 (1932). English translation by V.M. Volosov from a Russian translation by Uspensky, V.A.: On the interpretation of intuitionistic logic, Selected Works of Kolmogorov, A.N.: Mathematics and Mechanics, vol. 1, pp. 151-158 Kluwer, Dordrecht, (1991) 
18. Lambek, J., Scott, P.J.: Introduction to Higher Order Categorical Logic. Cambridge University Press, Cambridge (1986)

19. Rose, K.H.: Explicit substitution: tutorial and survey. In: BRICS Lecture Series. University of Aarhus, Aarhus (1996)

20. Schroeder-Heister, P.: Proof-theoretic versus model-theoretic consequence. In: Peliš, M. (ed.) The Logica Yearbook 2007, pp. 187-200. Prague (2008)

21. Schroeder-Heister, P.: The categorical and the hypothetical: a critique of some fundamental assumptions of standard semantics. In: Lindström, S. et al. (eds.) The Philosophy of Logical Consequence and Inference, Proceedings of the Workshop "The Philosophy of Logical Consequence, Uppsala, 2008”, Synthese, vol. 187, pp. 925-942 (2012)

22. Schroeder-Heister, P., Contu, P.: Folgerung. In: Spohn, W., et al. (eds.) Logik in der Philosophie, pp. 247-276. Synchron, Heidelberg (2005)

23. Simpson, J., Weiner, E. (eds.): The Oxford English Dictionary, 2nd edn. Oxford University Press, Oxford (1989)

24. Vranas, P.B.M.: In defense of imperative inference. J. Philos. Log. 39, 59-71 (2010)

25. Wittgenstein, L.: Logisch-philosophische Abhandlung. Annalen der Naturphilosophie 14, 185262 (1921). English translation by Ogden, C.K.: Tractatus logico-philosophicus, Routledge, London (1922) new translation by Pears D.F., McGuinness B.F., Routledge, London, (1961)

26. Wittgenstein, L.: Philosophische Untersuchungen. Blackwell, Oxford (1953). English translation by Anscombe, G.E.M.: Philosophical Investigations, 4th edn. with revisions by Hacker P.M.S., Schulte J., Wiley-Blackwell, Oxford (2009)

27. Wittgenstein, L.: Wittgenstein's Lectures, Cambridge 1932-1935. In: Ambrose, A. (ed.) From the Notes of Alice Ambrose and Margaret Macdonald. Blackwell, Oxford (1979) 